\title{
Causes and management of bronchiolitis with chronic obstructive features
}

\author{
I G C HODGES, A D MILNER, R C GROGGINS, AND G M STOKES \\ Department of Child Health, University Hospital, Queen's Medical Centre, Nottingham
}

SUMMARY During the last 4 years we have seen 13 children with a severe bronchiolitis. The children have had prolonged respiratory illnesses waxing and waning over many months, often requiring oxygen therapy for long periods. The diagnosis is based on a clinical picture similar to acute bronchiolitis, but persisting for months, frequently associated with failure to thrive, coupled with lung function evidence of hyperinflation and airways obstruction but normal static compliance. Confusion with "other chronic lung conditions can be clarified by straightforward observations and investigations. We believe that chronic bronchiolitis is fairly common and often misdiagnosed. The short- and medium-term prognosis appears good although small airways disease may persist into adult life.

The pattern of acute viral bronchiolitis with coughing, tachypnoea, hyperinflation, and widespread crepitations continuing for up to 2 weeks is familiar to all paediatricians. On occasions this clinical picture can persist for many months and is then due to more extensive viral lung damage. ${ }^{1}$ Most cases previously reported are due to adenoviral infections, particularly types 3,7 , and $21,{ }^{1-3}$ rather than to respiratory syncytial virus, and in some, specimens at necropsy have shown an obliterative bronchiolitis. ${ }^{4}$ This condition is considered to be rare. During the last 4 years we have seen 13 children with the identical clinical features, but in whom the aetiology could often not be established. Although they probably represent a heterogeneous group, their presentation, results of lung function tests, and outcome indicate that from the practical point of view they can be considered as a single clinical entity. In this paper we wish to describe these children, providing information on the mode of presentation, findings on examination, and results of investigations including lung function tests and subsequent progress.

\section{Mode of presentation}

The mean age of the 13 children at the time of the initial illness was 9 months (range 7 weeks to 3 years 9 months); however 4 were over 12 months. Five of the children had first-degree relatives with asthma. The illness was acute in onset in 11 children, although the average duration of symptoms before hospital referral was 5 weeks. Six children had been unwell with respiratory symptoms for longer than a month at the time of first hospital referral.

The two most common symptoms were rapid breathing which interfered with feeding, and coughing day and night, often severe enough to produce repeated vomiting.

The illness started in the months of December to March in only 4, in October or November in a further 4, and in May and July in the remainder.

\section{Clinical features}

The most consistent findings when initially referred to hospital were hyperinflation of the chest with associated hepatomegaly, tachypnoea (respiratory rate $>60 / \mathrm{min}$ ), and widespread crepitations. Wheezing was recorded in only 4 children. Birthweight and admission weight centiles were available on 11 children. Ten children were failing to thrive with 6 having weights below the 3 rd centile. The longer the duration of symptoms before admission the further the child was below his birthweight centile.

The initial diagnoses considered were acute bronchiolitis (3), asthma (1), cystic fibrosis (4), bronchopneumonia (2), fibrosing alveolitis (2), and pneumocystisis (1) .

\section{Subsequent progress}

Twelve of the 13 children were admitted to hospital. After admission the condition of all deteriorated 
except one child who had already been ill for 4 months at home. The mean duration of hospital stay was 61 (range 14 to 133) days. Eleven children received oxygen therapy. One required oxygen for 522 days, until he died at home. Excluding this child the mean duration of oxygen therapy was 25 days. Four children were treated by mechanical ventilation, one child being ventilated for a total of 20 days. He had the most widespread lung damage and was the child who eventually died in chronic respiratory failure. Three children were considered to be in heart failure as judged by apparent hepatomegaly, lung crepitations, and engorged neck veins. In retrospect, the hepatomegaly was almost certainly secondary to the hyperinflation, the crepitations due to bronchiolar infection and damage, and the engorged neck veins the result of high intrathoracic pressures impeding venous return to the heart.

\section{Chest x-ray findings}

At the initial presentation the outstanding features were hyperinflation, fine nodular opacities, and streaking radiating from the hilar region, mainly affecting the upper lobes. Two children had collapse or consolidation of the right middle lobe, 2 had areas of consolidation in the left lower lobe, and 1 had consolidation of the right upper lobe. The heart size was normal on all of the initial chest $x$-ray films. During the initial illness 4 children developed areas of consolidation that were not present on admission. In 2 the left lung subsequently became more hyperinflated and herniated across to the right hemithorax. In both children the breath sounds were diminished on the affected side, confirming the presence of MacLeod's syndrome. ${ }^{56}$

\section{Investigations}

Positive evidence of viral infection was found in only 3 cases. Two children had positive viral cultures and rising complement fixation titres for an adenovirus. One was grouped as a type 7 adenovirus. A further child had a rising complement fixation titre to Mycoplasma pneumoniae, the titre rising to 1 in 32 . All had negative responses to Mantoux and sweat tests. Alpha-1 antitrypsin levels and barium swallows were normal in the 8 and 7 children studied respectively. Serum immunoglobulin tests were unremarkable in 11 children but one had increased levels of IgG and IgM. Two children underwent cardiac catheterisation and were found to have normal hearts. One infant had a needle aspiration of the lung performed to identify the infecting agent and 3 underwent bronchoscopy. The only abnormal bronchoscopic finding was the presence of excessive amounts of white mucus.

\section{Lung biopsy}

Open lung biopsy was performed on 2 patients, neither of whom had been ventilated. A specimen at necropsy was from a third child who had been ventilated. One biopsy obtained 5 months after the onset of symptoms showed small round cells and the occasional eosinophil surrounding and infiltrating the walls of the bronchioles producing a degree of obstruction. Alveolar macrophages were prominent. She is now symptom free 4 years after the initial illness.

In the other biopsy taken 4 months after the onset of the illness there was a round cell infiltrate, marked loss of cilia, and some bronchioles obliterated by organising vascular fibrous tissue. There were areas of collapse with adjacent areas of compensatory overinflation. The specimen at necropsy demonstrated in addition to the cellular infiltrate and obliteration, areas of gross overdistention, with remodelling of the lung architecture in an abnormal manner.

\section{Lung function}

All but one child who was critically ill had at least one lung function assessment. Thoracic gas volume was measured by plethysmography 78 and total respiratory resistance by the forced oscillation technique. ${ }^{910}$ Both measurements necessitate prior sedation with chloral hydrate $(80$ to $100 \mathrm{mg} / \mathrm{kg})$. All the children were hyperinflated ${ }^{11}$ when first assessed (Table) with varying degrees of airways obstruction. Repeat lung function tests have been performed on 10 children (Table). Eight still have increased total respiratory resistance (B Taylor, 1975, unpublished data). None showed an improvement of more than $10 \%$ after nebulised salbutamol $(0.5 \mathrm{ml}$ of $0.5 \%$ mass/vol solution), but one child had a $50 \%$ improvement in airways obstruction after inhaling a nebulised solution containing $250 \mu \mathrm{g}$ ipratropium bromide. Four children have been given oral steroids, 2 with lung function monitoring.

Table Results of initial and latest thoracic gas volume and total respiratory resistance measurements as a percentage of expected

\begin{tabular}{lllll}
\hline Assessment & $\begin{array}{l}\text { Time } \\
\text { between } \\
\text { illness and } \\
\text { assessment } \\
\text { (months) }\end{array}$ & $\begin{array}{l}\text { Results as a percentage } \\
\text { of expected }\end{array}$ & No tested \\
\cline { 4 - 5 } & $\begin{array}{l}\text { Thoracic } \\
\text { gas volume }\end{array}$ & $\begin{array}{l}\text { Total } \\
\text { respiratory } \\
\text { resistance }\end{array}$ & \\
\hline $\begin{array}{l}\text { First } \\
\text { Mean }\end{array}$ & $11 \cdot 8$ & 206 & 169 & 12 \\
$\begin{array}{c}\text { Range } \\
\text { Latest } \\
\text { Mean }\end{array}$ & $1.5-36$ & $140-298$ & $85-250$ & \\
Range & 33.9 & 194 & 191 & 10 \\
\hline
\end{tabular}


In these 2 there was no improvement in lung function after one week of prednisolone $(2 \mathrm{mg} / \mathrm{kg}$ per 24 hours). One child had been given increasing doses of prednisolone for many months because of an apparent initial clinical improvement, resulting in a grossly cushingoid and stunted appearance. His growth has subsequently improved after the reduction in oral steroids. The remaining child showed no clinical improvement after a short course of steroids.

Static compliance measurements were performed on 7 children using a respiratory jacket which surrounded the chest and abdomen. The jacket was inflated to a pressure of $3.5 \mathrm{~cm}$ of water and connected to a pressure transducer. The jacket was calibrated by injecting and withdrawing known volumes of air with a $50 \mathrm{ml}$ syringe, and measuring the shift in end tidal baseline. ${ }^{12} \mathrm{~A}$ 2-way tape mounted on a soft-flanged face mask allowed us to connect the child to a pre-pressurised 100-litre tank for several breaths, thus providing varying amounts of continuous positive airways pressure. Relating the applied pressure to the induced changes in end tidal baseline as measured by the jacket allowed us to derive the pressure volume relationship of the total respiratory system and hence the static compliance in each child up to age 25 months. All the measurements were within the normal range (B Taylor, 1975, unpublished data).

\section{Outcome}

Eight children still have troublesome symptoms between 2 months and 6 years after the onset of the illness. All have made progress although improvement has been slow. The first sign of improvement was reflected by remission of their failure to thrive, regaining the 3rd centile for weight by 3-24 months after the onset of the illness. Five have been followed up for longer than a year. All have had repeated hospital admissions for respiratory tract problems. The most striking finding on follow-up examination was the hyperinflation. Crepitations tended to disappear between 2 and 24 months after the original illness, but returned when the child had a superimposed respiratory tract infection. Finger clubbing developed in only one child who died in respiratory failure. The 3 children who have been followed up for less than one year all still have persistent crepitations and hyperinflation. Only one child has normal lung function and is asymptomatic. Of the remaining 4 , one child aged $4 \frac{1}{2}$ years has only an intermittent cough. The other 3 are asymptomatic 18 months, 4 years 2 months, and 5 years 7 months after the onset of the illness. One of the 3 remains hyperinflated 5 years 7 months after the initial illness while another has normal lung function at 18 months. This is the only child who now has an entirely normal chest $x$-ray film without hyperinflation and streaky shadows.

Of those who were treated with intermittent positive pressure ventilation one child, the most severely affected, died at home 19 months after the initial infection. He had a confirmed adenoviral bronchiolitis and had been entirely oxygen dependent, requiring between 50 and $100 \%$ oxygen in the last 3 months of his life. Only one of the remaining 3 children is symptom free.

\section{Discussion}

All 13 children were referred as diagnostic problems, either by general practitioners, or by paediatricians elsewhere. All had severe chronic lung disease with persistent coughing and often vomiting, respiratory distress, and failure to thrive. The clinical picture was that of acute bronchiolitis with tachypnoea, recession, hyperinflation, and widespread crepitations which persist for months. A variety of provisional diagnoses had been made, including fibrosing alveolitis, bronchopneumonia, and cystic fibrosis. Clinically they most closely resembled obliterative bronchiolitis, a condition in which there is widespread persisting damage to the small airways, usually resulting from a severe viral infection, or from an adenovirus. The clinical findings, hyperinflation on chest $x$-ray film, and results of lung function tests, leave us in no doubt that the major site of damage in this group of children is also in the small airways. This has been confirmed by the 2 lung biopsies and the single specimen at necropsy. However, we have evidence of adenoviral infection in only 2 of the group. We suspect that this clinical picture of chronic bronchiolitis represents the response of the lung to a number of different pathological processes, and so has to be considered as a heterogeneous group. Nevertheless, from the investigatory, management, and prognostic point of view, they could be considered together.

\section{Investigation}

The main aim in investigating these children is to establish that the site of damage is primarily in the small airways and that other clinical entities have been excluded. Chest $x$-ray films supported the clinical evidence of hyperinflation and showed a linear streaking rather than diffuse or hilar granular shadow, making it very likely that the site of damage is in the medium or small airways rather than in the lung parenchyma. Lung function tests confirmed this, providing evidence that the children were 
indeed grossly hyperinflated with airways obstruction and yet had normally compliant lungs.

The condition with which the clinical picture of these children most closely corresponds is cystic fibrosis, despite the absence of pancreatic involvement. For this reason, at least one sweat test is required. This has been entirely normal in all our children. Combined immunological deficiency state can also produce failure to thrive with chronic respiratory disease and must be excluded by examining the child for the presence of lymphatic tissues and measuring at least one differential white cell count and levels of serum immunoglobulins. Again, all measurements, except one that showed raised levels of IgM and IgG, were normal. Pharyngeal incoordination, oesophageal reflux, or an H-type tracheo-oesophageal fistula may also lead to hyperinflation and chronic respiratory symptoms. These conditions can be excluded by cine swallow and meal if there is any doubt about the diagnosis. Further support can be gained by examining tracheal aspirate for fat-laden macrophages. In our children, there was no evidence that aspiration was occurring.

Alpha-1-antitrypsin deficiency has not been reported as a cause of lung disease in this age group, but is worth excluding, as chronic bronchitis and emphysema can undoubtedly occur in late childhood and early adult life. The immotile cilia syndrome, although a cause of chronic respiratory disease, is not likely to be confused as it causes bronchiectasis and parenchymous lung disease rather than hyperinflation, but can be excluded by light and electron microscopical examination of respiratory tract epithelial cells obtained from nasal or tracheal biopsy.

An initial diagnosis of fibrosing alveolitis ${ }^{13}$ was made on 2 of our group. This condition, which presents with cough, tachypnoea, failure to thrive, and persistent fine crepitations can easily be confused with chronic bronchiolitis. The main distinguishing features are the presence of finger clubbing, chest $x$-rays films showing diffuse and later discrete ${ }^{13}$ shadows, and reduced lung compliance; none of which was present in these children.

In one child lung biopsy had been performed before transfer to Nottingham. The other was obtained at a time when we had only seen one previous case, and were unsure of the diagnosis. Unfortunately, needle biopsy is not likely to provide sufficient tissue, and may well lead to pneumothorax in the presence of severe airways obstruction. Thus an adequate lung biopsy necessitates thoracotomy, an invasive procedure in a child who already has severe respiratory problems. We would now only proceed to open lung biopsy in a child with this clinical picture if there was progressive deterioration.

\section{Management}

The management in the short-term consists of oxygen therapy to relieve hypoxia, antibiotics for secondary infection, regular physiotherapy, and if necessary feeds via nasogastric tubes. The children can be discharged home once they are no longer oxygen dependent and are feeding satisfactorily. They are likely still to have little respiratory reserve with tachypnoea, hyperinflation, and widespread crepitations. There is no evidence that bronchodilator drugs or corticosteroids have any effect, but both are probably worth trying for 2 or 3 weeks. If there is no obvious benefit, the steroids should be stopped. One of our children had total growth suppression as a result of high dosages of steroids during a period of 15 months, which had not had any apparent effect on the underlying condition. The children will continue to require physiotherapy from their parents as lung clearance mechanisms are undoubtedly faulty. They will need courses of broad-spectrum antibiotics for superimposed infection. We have also tended to give prophylactic antibiotics (co-trimoxazole) in the winter to any child who has had more than one infection in a period of about 8 weeks.

\section{Prognosis}

At the height of the illness these children were very ill, with severe respiratory distress and failure to thrive. Most parents had been given a very guarded prognosis, which inevitably caused a great deal of anxiety. Our experience indicates that the longer term outlook is surprisingly good. Only the one child who required ventilation for 25 days died, the others showing resolution of their failure to thrive after 3 to 24 months. This improvement probably reflects new lung growth. Inevitably, these children have very little respiratory reserve, and are likely to require further hospital admissions, particularly in the winter months, for superimposed infection. Nevertheless, an optimisitic prognosis should be given to the parents as the outlook in the next months and years is good. We do not yet know whether respiratory problems will recur in adult life.

We thank the general practitioners and senior paediatricians who referred these children for investigation, and also the Asthma Research Council for their continued financial support.

\section{References \\ 1 Wohl M E B, Chernick V. Bronchiolitis. Am Rev Respir Dis 1978; 118: 759-81.}


${ }^{2}$ Lang W R, Howden C W, Laws J, Burton J F. Bronchopneumonia with serious sequelae in children with evidence of adenovirus type 21 infection. $\mathrm{Br} \mathrm{Med} J 1969$; i: 73-9.

3 Steen-Johnsen J, Orstavik I, Attramadal A. Severe illness due to adenovirus type 7 in children. Acta Paediatr Scand 1969; 58: 157-63.

- Becroft D M O. Bronchiolitis obliterans, bronchiectasis, and other sequelae of adenovirus type 21 infection in young children. J Clin Pathol 1971; 24: 72-82.

5 MacLeod W M. Abnormal transradiancy of one lung. Thorax 1954; 9: 147-53.

- Cumming G R, MacPherson R I, Chernick V. Unilateral hyperlucent lung syndrome in children. $J$ Pediatr 1971; 78: $250-60$.

7 Radford M. Measurement of airway resistance and thoracic gas volume in infancy. Arch Dis Child 1974; 49: 611-5.

8 Stocks J, Levy N M, Godfrey S. A new apparatus for the accurate measurement of airway resistance in infancy. J Appl Physiol 1977; 43: 155-9.

9 Dubois A B, Brody A W, Lewis D H, Burgess B F, Jr.
Oscillation mechanics of lungs and chest in man. $J$ Appl Physiol 1956; 8: 587-94.

10 Cogswell J J. Forced oscillation technique for determination of resistance to breathing in children. Arch Dis Child 1973; 48: 259-66.

11 Doershuk C F, Downs T D, Matthews L W, Lough M D. A method for ventilatory measurements in subjects one month to five years of age: normal results and observations in disease. Pediatr Res 1970; 4: 165-74.

12 Stokes G M, Milner A D, Johnson F, Hodges I G C, Groggins R C. Measurement of work of breathing in infancy. Pediatr Res $1981 ; 15$ : 22-7.

13 Hewitt C J, Hull D, Keeling J W. Fibrosing alveolitis in infancy and childhood. Arch Dis Child 1977; 52: 22-37.

Correspondence to Professor A D Milner, Child Health Department, E Floor, East Block, University Hospital, Queen's Medical Centre, Derby Road, Nottingham NG7 2UH.

Received 12 March 1982 